Marek Bistak - Stefan Medvecky - Eva Gajdosova - Marian Dzimko - Stanislav Gramblicka

Robert Kohar - Marian Stopka - Jan Steininger - Slavomir Hrcek - Michal Tropp - Frantisek Brumercik*

\title{
APPLICATIONS OF MODERN TECHNOLOGIES IN THE PRODUCTION OF AIRCRAFT PROPELLER PROTOTYPE
}

In the field of engineering relatively new designs and manufacturing processes are constantly emerging. These changes are often seen in designing and manufacturing of prototypes or in single part production. We decided to analyse an aircraft propeller prototype using modern technologies. Existing aircraft blade design was converted to digital models which were analysed.

Keywords: 3D scanning, 3D printing, composite, aircraft propeller, analysis.

\section{Introduction}

Aircraft propellers and their applications have been known for many years. The first propellers were already used at the time of ancient Greece for pumping water, known as the Archimedes' screw. Over time they were improved and applied in the new areas of application, for example, in aircraft engineering. A typical material of propeller blades is wood, but nowadays laminated plastic or carbon fibres are more commonly used.

These materials are costly and time consuming in prototype production. We decided to use modern technologies and ways to improve production parameters by manufacturing functional prototypes with a view of price and complicacy of production.

The existing aircraft propeller blade design was converted to a digital form. Then the propeller hub for fixing of 3 blades was modelled. Dynamic and stress analysis was progressively done. Finally were compared the costs of $3 \mathrm{D}$ printing and conventional machining.

\section{3D Laser Scanning}

The existing aircraft propeller blade was used to perform the analysis. The 3D model was created in the first step for the further computer processing. An appropriate way to achieve that is a $3 \mathrm{D}$ scanning. In this case we used a Faro Laser Line probe. It's a non-contact laser measuring and scanning system. Points are measured by combining a laser beam and special digital chip.
This technology allows scanning speed of more than 19000 points per second. The accuracy of the scanner is $0.035 \mathrm{~mm}$ but the final result of scanning depends on the used measuring arm. The resulting accuracy is the sum of accuracies of the measuring arm and the scanner. The Laser Line Probe V3 specifications are in Table 1.

Specification of 3D laser scanner [1] Table 1

\begin{tabular}{|l|l|}
\hline Accuracy & $\pm 35 \mu \mathrm{m}$ \\
\hline Repeatability & $35 \mu \mathrm{m}$ \\
\hline Minimal measuring distance & $95 \mathrm{~mm}$ \\
\hline Optimal measuring distance & $95 \mathrm{~mm}$ to $180 \mathrm{~mm}$ \\
\hline Scanner spread & $34 \mathrm{~mm}$ to $60 \mathrm{~mm}$ \\
\hline Points per frame & 640 points on frame \\
\hline Measurement frequency & 30 frames per second \\
\hline Laser type & $660 \mathrm{~nm}$, CDR class II/IEC class $2 \mathrm{M}$ \\
\hline Weight (no arm) & $370 \mathrm{~g}$ \\
\hline
\end{tabular}

Before scanning a thin layer of chalk flour was applied. The blade was painted because reflections on the surface could cause problems to the laser scanner system. The scanning blade can be seen in Fig. 1.

After the propeller blade scanning it was found out that the model created by scanning was not suitable for the finite element method analysis, because the created model mesh did not have a good interconnection between the nodes. Therefore, it was necessary to create a 3D model in the CAD system. The sections

\footnotetext{
* Marek Bistak, Stefan Medvecky, Eva Gajdosova, Marian Dzimko, Stanislav Gramblicka, Robert Kohar, Marian Stopka, Jan Steininger, Slavomir Hrcek, Michal Tropp, Frantisek Brumercik

Department of Design and Mechanical Elements, Faculty of Mechanical Engineering, University of Zilina, Slovakia

E-mail: marek.bistak@fstroj.uniza.sk
} 
from the scanned model were used and then connected by using the tool "sweep". The 3D model of blade is in Fig. 2.

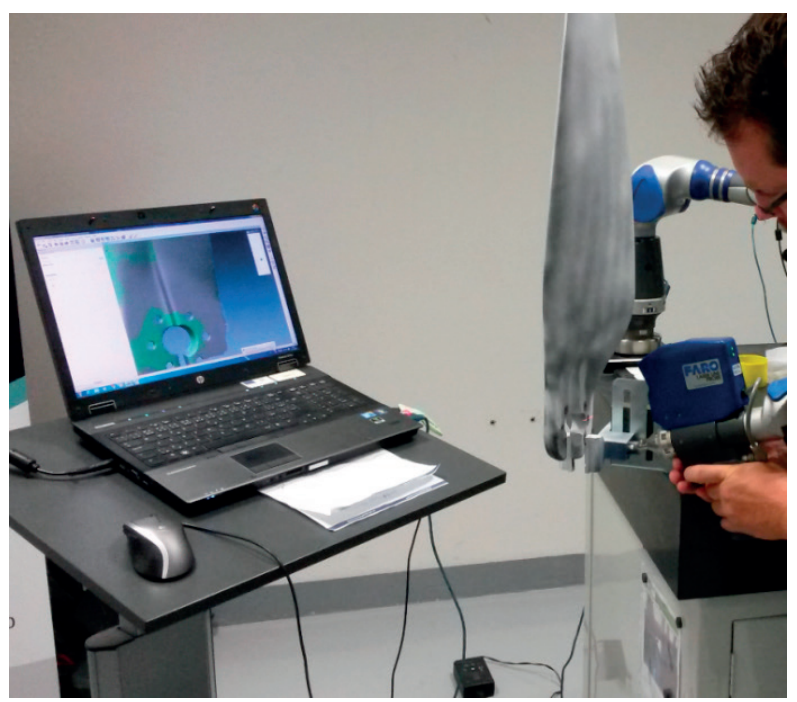

Fig. 1 Blade scanning by FARO Laser Line Probe

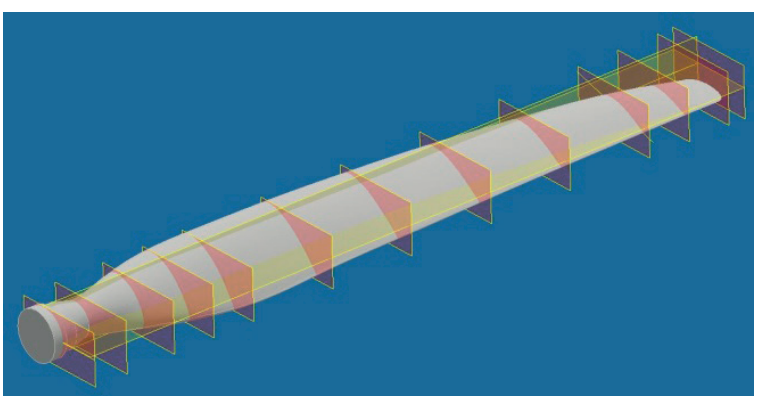

Fig. 2 Model of propeller blade designed by tool "sweep“

It was necessary to design a 3 blade propeller hub for the finite element method analysis. The propeller hub, which is seen in Fig. 3, was designed in the CAD program, but no canning was used [2].

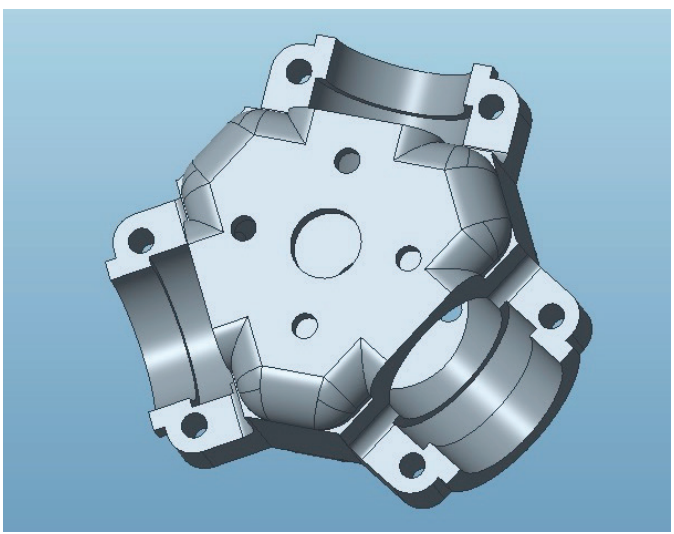

Fig. 3 Three-blade propeller hub designed in PTC CREO PARAMETRIC 3.0

\section{Calculation of centrifugal force}

After designing the 3D model it was necessary to investigate the loads acting on the propeller. Pulling force on one propeller blade was measured in real conditions and the value is 1000 $N$. The task was to calculate the centrifugal force value of one propeller blade during the performance of rotary movement for the purpose of verifying the correctness of the design.

Input parameters:

$R=323.5 \mathrm{~mm}$ (distance between the centre of gravity of the propeller blade and hub axis), $m=0.904 \mathrm{~kg}$ (weight of one propeller blade), $n_{l}=3000 \mathrm{rpm}$ (maximal constant propeller speed).

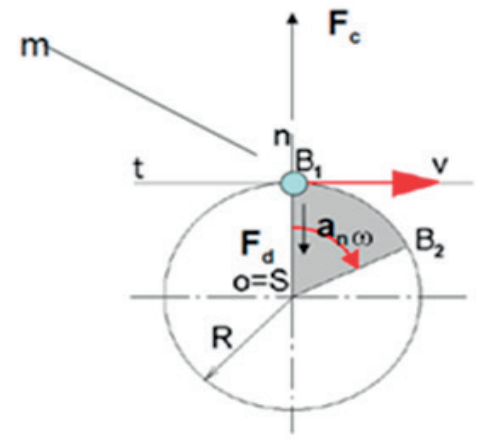

\section{Legenda}

$F_{c}-$ centrifugal force

$\mathbf{F}_{\mathrm{d}}$ - centripetal force

$\mathbf{a}_{\mathrm{n}}$ - normal acceleration of aircraft blade

$\mathrm{S}$ - centre of fixed rotation axis

$\mathbf{R}$ - distance between center of gravity and center of rotation (radius)

$\boldsymbol{\omega}$ - angular velocity

$\mathbf{B}_{3}$ - gravity centre of aircraft blade (mass point)

$\mathbf{B}_{2}$ - gravity centre of aircraft blade in motion

m- aircraft blade weight

Fig. 4 Force analysis of the dynamics centre of mass around fixed rotation axis

Uniform rotary motion is defined as a rotation motion of the point mass around a fixed axis at constant speeds. As a result of that the centrifugal force is acting to a point mass, as a reaction to centripetal force (Fig. 4). To enable the motion of a point mass on a circle, it is necessary that centripetal force constantly determine the still normal acceleration into the middle of the movement. During the uniform rotary motion of the point the circumferential speed is continuously changing direction by uniform rotary motion and gradually turns to the centre of rotation. This shows that rotating point mass continuously increases the speed into the middle of circle and therefore during the rotation motion of point, its determined direction into the middle of acceleration is 
called centripetal or normal acceleration $a_{n}$, because it is acting in direction of the normal motion [3].

Angular velocity calculation

$\omega=2 \pi \cdot n=2 \pi \cdot \frac{3000}{60}=314.16 \mathrm{rad} \cdot \mathrm{s}^{-1}$

Normal acceleration calculation

$a_{n}=R \cdot \omega^{2}=0.3235 \cdot 314.16^{2}=31928.17 m \cdot s^{-2}$

Centrifugal force calculation

$$
F_{c}=m \cdot a_{n}=0.904 \cdot 31928.17=28862.4658 N
$$

Verification of the analytical calculation of centrifugal force was done in the MSC ADAMS program. The functional kinematic model consisting of the fixed rotation axis and point mass was designed. The weight and the exact distance from the fixed rotation axis were predefined to a point mass. The rotatory constrain with a defined rotation speed was attached to the place of the axis of rotation. Consequently, the simulation was done with 50 steps and time 0.1 second. After performing the simulation, the centrifugal force characteristic was obtained [4].

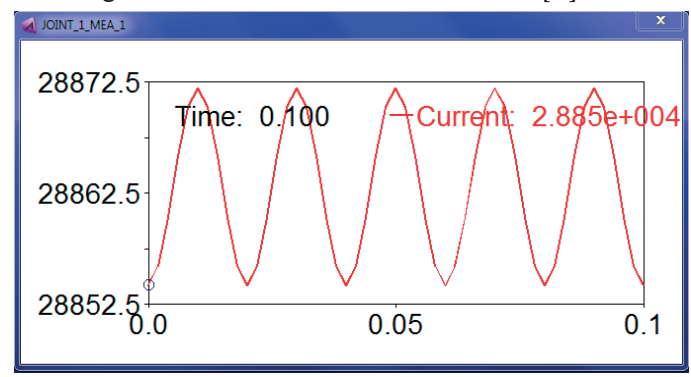

Fig. 5 Centrifugal force characteristic

Simulation in MSC Adams confirmed the accuracy of analytical calculation and displayed graphic progress of centrifugal force on one propeller blade (Fig. 5). We confirmed the correctness of the design. Calculated centrifugal force is $28862.5 \mathrm{~N}$

\section{Strength calculation of aircraft propeller blade made of composite material}

This part of the article describes the strength calculation process in ANSYS Workbench program. To obtain the load stress value and the component deformation it is necessary to follow the next steps:

- 3D model design: The real blade is hollow, so the blade model design was done as the shell model in Creo Parametric. The shell model was created as a coating surface of the solid mode.

- Defining of boundary conditions: The boundary conditions of the component were defined in Ansys Workbench - value and location of the applied load and location of the number of degrees of freedom removal (Fig. 6).

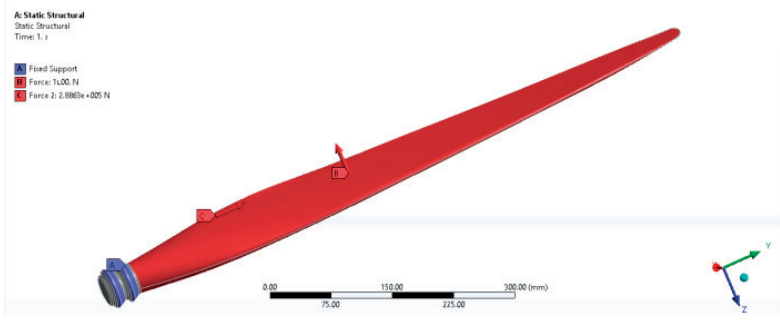

Fig. 6 Defining of boundary conditions

- Material defining: The component is made of carbon fibre composite. From the library we selected the material whose characteristics correspond to impregnated carbon fabric. The next step was to create a fabric with a thickness corresponding to the thickness of the fabric carbon offered by different manufacturers. After defining the type and thickness, the creation of fibre-glass is performed (Fig. 7). It's very important to correctly choose the order of layers and the orientation of the carbon fibres.

Figure 7 shows distribution of the fibres in the fibre-glass (composite) and directional toughness which depends on the angle of rotation of the individual layers towards the neutral axis. Value $a$ is the fabric turning towards the neutral axis expressed in degrees. Value $t$ is the thickness of each layer expressed in meters [5].

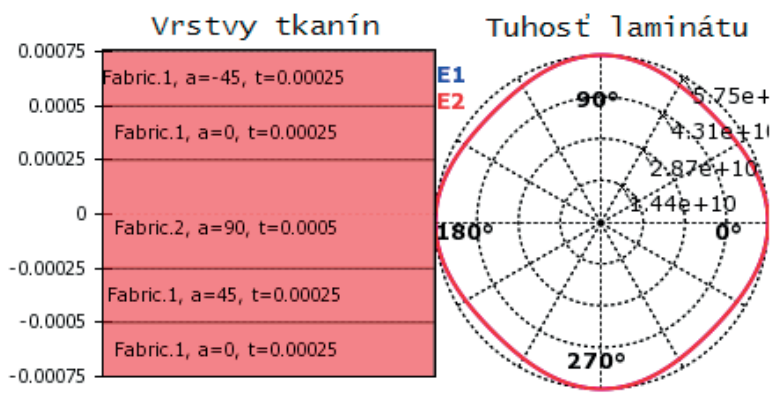

Fig. 7 Fibre-glass characteristic [6]

- Selection of the fibre-glass layers orientation: After entering the properties of fibre-glass layers, it's necessary to select a surface, which defines the shape and neutral axis of orientation of single fibres. 


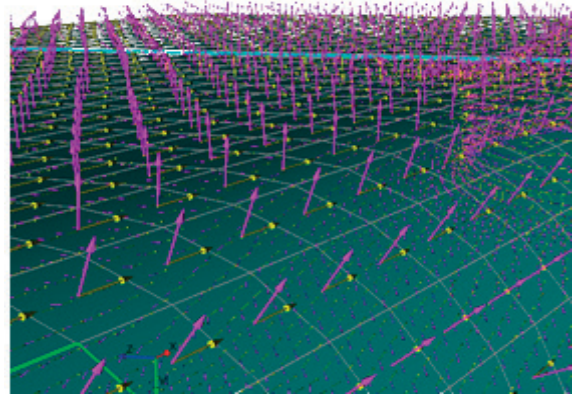

Fig. 8 Laminate layers orientation

Figure 8 represents directional vectors needed for packaging the selected area. The yellow arrow shows neutral axis of individual fibre-glass layers, the purple arrow shows normal direction of layers lying in regard to the chosen surface. After defining the material and the boundary conditions it was possible to finish the calculation. Entry centrifugal force is $28862.5 \mathrm{~N}$, pulling force is $1000 \mathrm{~N}$ and the tension in material may not exceed $400 \mathrm{MPa}$ by a glass laminate and 600MPa by a carbon laminate [7].

The calculated maximal aircraft blade tension is $108 \mathrm{MPa}$, so it meets the given criteria. Figure 9 shows tension behaviour. Interesting information is the maximal bending. Bending behaviour is shown in Fig. 10. At the end of the carbon laminate blade there is a $30 \mathrm{~mm}$ bend.

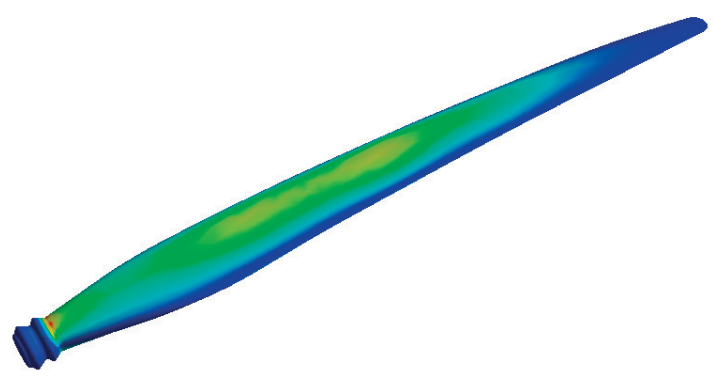

Fig. 9 Material tension (von Mises)

Maximal bending by aircraft glass laminate is $48 \mathrm{~mm}$ at the end of the blade. This value is $12 \mathrm{~mm}$ higher than the value by carbon laminate, exactly $60 \%$ higher.

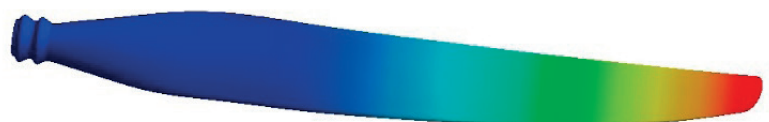

Fig. 10 Total deformation

Generally, the carbon laminate has more advantages than glass laminate. The main advantage is decreasing the blade weight from $904 \mathrm{~g}$ to $76 \mathrm{lg}$. That means lower centrifugal forces and lower load stress of the blade propeller hub. In case of using higher strength carbon material, it's possible to decrease even more the weight and centrifugal forces. Higher stiffness is profitable, to ensure the stability under load and for better aerodynamic properties. Disadvantages are complicated production and higher costs. For verification of the function the fibre-glass laminate is sufficient for prototype production. For practical production the carbon fibre parts are more suitable [8].

\section{Strength analysis of the blade propeller hub made of aluminium alloy}

The aim of this FEM analysis is to compare the hub mechanical properties produced by conventional machining of EN AW 7075 (AlZnMgCu1.5). This material is applied in aircraft industry with AlSi10Mg used in 3D printing. The analyses were calculated in ANSYS Workbench (Fig. 11). In case the 3D printed blade propeller hub has the required parameters, the 3D printing may also be used in practice. That means a shorter production process and more solutions for designers. Table 2 shows the basic material properties.

Material properties

Table 2

\begin{tabular}{|ll|c|c|}
\hline Material & AlZnMgCu1.5 & AlSi10Mg \\
\hline Description & \multicolumn{2}{|c|}{ Dimension } \\
\hline Material density & $\rho, \mathrm{kg} . \mathrm{m}^{-3}$ & 2810 & 2680 \\
\hline Tensile strength & $\mathrm{R}_{\mathrm{m}}, \mathrm{MPa}$ & 460 & 460 \\
\hline Yield strength & $\mathrm{R}_{\mathrm{p} 0,2}, \mathrm{MPa}$ & 360 & 270 \\
\hline Young's modulus & $\mathrm{E}, \mathrm{GPa}$ & 72 & 75 \\
\hline Tensility & $\mathrm{A}, \%$ & 6 & 9 \\
\hline
\end{tabular}

For the FEM analysis the 3D model had to be prepared. At first, the assembly was created from the hub and three holders. The blades were not necessary for the analysis and were not mentioned. Instead of them the thin wall tubes were used. Another modification consisted of removing cosmetic features as threads [9].

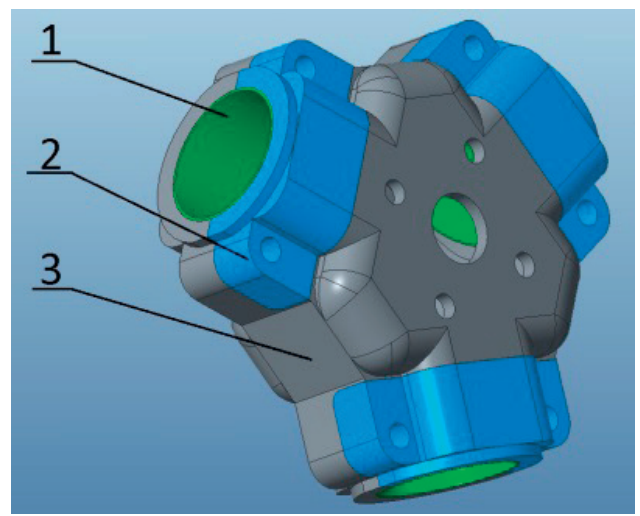

Fig. 11 3D model (1-tube, 2-holder, 3-hub) 
Having optimized the model, it was used for meshing. Then the Degrees of Freedom were removed by the function 'fixed support' applied to the surface, where the real hub is attached to the shaft flange. The loads were defined at last. Each blade was loaded by the pulling force $F t=1000 \mathrm{~N}$ by using of function "remote force" placed at a distance $a=323.5 \mathrm{~mm}$ from the axis. This distance corresponds to the current position of pulling force to the axis and creates the bending moment. The centrifugal force of each blade was defined at the same time. Size dimension of this force was calculated in Chapter 3. The preparatory part was done and the calculation was executed. Development of stresses and deformations were equivalent in regard of the same model shape and same loads, but the resultant values were different. The deformation characteristic is shown in Fig. 12 (left) and stresses characteristic (right).

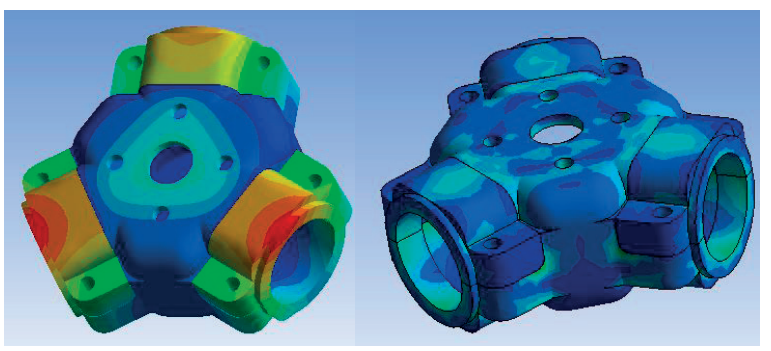

Fig. 12 Deformation characteristic (left) and stress characteristic (right)

The maximal calculated stress in the component made of $\mathrm{AlZnMgCu} 1.5$ was $173.86 \mathrm{MPa}$. This stress was created at the connection between the hub and blade, where line contact occurred. The rest of the hub was stressed up 60MPa. The maximum total deformation was $0.241 \mathrm{~mm}$ in the place, where the part absorbs the bending moment of pulling force. In this case the material should transfer the loads, because the stresses are under $R m$ and $R p 0.2$. By using 3D printing the maximal stress was calculated to $163.48 \mathrm{MPa}$ at the same places as before. The rest of the stresses were about $60 \mathrm{MPa}$. The maximal deformation $0.2339 \mathrm{~mm}$ was calculated on the holder. Again, the component should transfer the loads. The value of deformations and stress characteristic can be influenced by appropriate design modification, but due to the calculated results it is not necessary. It can be said generally that in this case the $3 \mathrm{D}$ printing can be used to produce a functional prototype of hub.

\section{Economic balance}

Results from the previous chapter are useful to compare the conventional production and $3 \mathrm{D}$ printing. In conventional production we have to consider material price, distribution and then materials needed for the desired shape [10]. The material price for EN AW 7075 ( $\mathrm{AlZnMgCu} 1.5)$ is $5.95 € / \mathrm{kg}$ including cutting. The price was determined on the supplier's offer. The weight of the workpiece for the hub is $7.868 \mathrm{~kg}$, then the price is $46.8 €$. The holder weight is $0.562 \mathrm{~kg}$; the price is $3.43 €$. The total price consists of one piece of hub and 3 pieces of holders; that is $48.49 €$. Another item in the production is the price of machining. That price was determined from the average of 3 independent price offers. The price offers are in Table 3.

Conventional production

Table 3

\begin{tabular}{|l|l|l|l|l|}
\hline Offer & $\mathbf{1}$ & $\mathbf{2}$ & $\mathbf{3}$ & Average \\
\hline Hub & $120.8 €$ & $200 €$ & $2500 €$ & $940 €$ \\
\hline Holder & $48.8 €$ & $36 €$ & $300 €$ & $128.3 €$ \\
\hline
\end{tabular}

The costs for machining are $1324.9 €$. The total price of material with the price for machining (hub and 3 holders) is 1373.4€. When AlSi10Mg is used for 3D printing it is necessary to calculate the cost of printing and machining functional surfaces. Due to the fact that $3 \mathrm{D}$ printing using sintered aluminium powder is not common we received only one price offer. The price of hub was $1143.6 €$ and the price of holder was $285.9 €$. The final price of printing is $2001.3 €$. For machining 3 price offers were received. Following Table 4 shows the price offers [11].

3D printed model machining

Table 4

\begin{tabular}{|l|l|l|l|l|}
\hline Offer & $\mathbf{1}$ & $\mathbf{2}$ & $\mathbf{3}$ & Average \\
\hline Hub & $91.7 €$ & $68 €$ & $750 €$ & $303 €$ \\
\hline Holder & $44.7 €$ & $16 €$ & $200 €$ & $86.9 €$ \\
\hline
\end{tabular}

The machining costs for $3 \mathrm{D}$ printing are $563.7 €$, which is $2.5 x$ less than conventional production. Total costs for printing and machining are $2565 €$. The machining costs and the total costs are compared in Fig. 13.

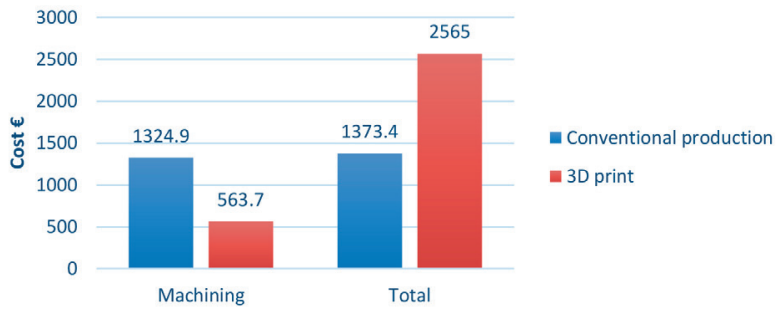

Fig. 13 Comparison of conventional production and 3D printed model machining cost

As we can see from the graph, in the case of classical machining the main part of the price is the machining (96\%), while in $3 \mathrm{D}$ printing it is only $22 \%$. The $3 \mathrm{D}$ model printing is significantly more expensive and the total price is almost twice higher than in the case of conventional machining. It can be said that conventional machining is still used in prototype production. The blank delivery time also increases the production process. 
Hence 3D printing is advantageous as the production does not need any preparation. In spite of the higher price, this method is often more preferred because the production is fast [12].

\section{Conclusion}

The aim of this work was to analyse the possibilities of modern technologies in the production of functional prototypes. An aircraft propeller blade was selected as a suitable object for analysis. A blade shape design was used from a real propeller and the hub was designed in the CAD program. The real blade design was converted to a digital model using 3D laser scanning. Then a solid model was created for analyses. It was necessary to investigate the material properties and loads. The pulling force was found out experimentally during the working conditions. Centrifugal force was calculated analytically and the result was verified by simulation in the Msc ADAMS program.

Investigated loads were used for loading the aircraft blade in ANSYS Workbench. The task of this simulation was to compare the stresses occurring in the blades made from two different materials- glass and carbon fibre. Mechanical properties spoke clearly in favour of the use of carbon composite, for its lower weight and higher strength. The disadvantage is economic difficulty; therefore the carbon prototype depends on its use.

Another task where the found forces were used was the hub strength analysis. The aim was to compare stress in the case that the hub was made by conventional machining and 3D printing. These two difficult methods provide sufficient mechanical properties for the functional prototype. The advantage of $3 \mathrm{D}$ printing is fast production and possibility to produce complicated shape components. Its disadvantage is the high production price and the need to be machined. Fast production is important in 3D printing. In the case of conventional machining, the price could be reduced by suitable technological designing.

Generally, current modern technologies offer to designers more new possibilities. Their advantage is fast and easy prototype production. The disadvantage is often only higher price. Using the advanced technologies for prototyping depends on a specific piece, its use and degree of technological optimisation for a specific type of production.

\section{Acknowledgments}

This paper presents results of work supported by the Slovak Scientific Grant Agency of the Slovak Republic under the project No. VEGA 1/0077/15.

The research is supported by the following institutions: Slovak Research and Development Agency under the contract no. APVV14-0508 - Development of new methods for the design of special large-size slewing rings.

\section{References}

[1] FARO Technologies UK Ltd.: Faro Arm manual. 2009.

[2] KOHAR, R., et al.: Numerical Analysis of Roller Bearing. Applied Computer Science, vol. 12, No. 1, 2016, 5-16.

[3] GONDA, J.: Dynamics for engineers (in Slovak), Bratislava : s.n., 1966.

[4] JEDLINSKI, L., et al.: Application of Vibration Signal in the Diagnosis of IC Engine Valve Clearance. J. of Vibroengineering, vol. 17, No. 1, 2015, 175-187.

[5] MARTIKAN, M., BRUMERCIK, F., BASTOVANSKY, R.: Development of Mechatronic Deformation System, Applied Mechanics and Materials, vol. 803, 2015, 173-178.

[6] FATURIK, L., et al.: Comaprison of Structural Design in High and Ultra-High Cycle Fatigue Regions, Transactions of FAMENA, vol 38, No. 4, 2014, 1-12.

[7] BRUMERCIK, F., LUKAC, M., KRZYWONOS, L.: Driveability Simulation of Vehicle with Variant Tire Properties, Communications - Scientific Letters of the University of Zilina, vol. 18, No. 2, 2016, 34-37.

[8] LAWRENCE, L.: ANSYS Workbench Tutorial Release 14.0. s.1. : University of Texas at Arlincton, 2013. ISBN 987-1-58503-754-4.

[9] MICIETA, B., BINASOVA, V., HALUSKA, M.: The Approaches of Advanced Industrial Engineering in Netx Generation Manufacturing Systems, Communications - Scientific Letters of the University of Zilina, vol. 16, No. 3A, 2014,

[10] CACO, M., et al.: Automatical E-frame - increasing utility value of AGV system (in Slovak), ProIN. 17, 2016, 1, 34-37.

[11] BRUMERCIK, F., DANKO, R.: Transport Application of Hybrid Simulaton. Communications - Scientific Letters of the University of Zilina, vol. 16, No. 2, 2014, 20-24.

[12] TROPP, M.: Non-conventional Materials and Drives in Constrution of Small Transport Vehicles (in Slovak), Diploma thesis, Zilina, 2015. 